\title{
Dime cómo te mueves y te diré qué expresas: una propuesta para trabajar los gestos en el aula de $\mathrm{E} / \mathrm{LE}$
}

GLORIA UCLÉS RAMADA

Universitat de València / Grupo Val.Es.Co.

Gloria.Ucles@uv.es

Resumen: En esta propuesta didáctica se trabajará en la enseñanza de gestos en español para alumnos de nivel B2. Específicamente nos centraremos en gestos ligados con acciones cotidianas y emociones. Para ello se presentan una serie de actividades en las que se emplean muestras audiovisuales reales y se hace uso de aplicaciones móviles para la puesta en práctica de los gestos por parte de los alumnos.

Palabras clave: pragmática, gestos, comunicación no verbal.

\section{A proposal to work with gestures in the Spanish as a foreign language classroom}

Abstract: This proposal deals with teaching gestures in Spanish for B2 level learners. Specifically, attention will be put on gestures that express everyday actions and emotions. To do so, we propose a set of exercises, in which clips extracted from real interactive situations are used to learn and recognise gestures, and mobile apps are deployed so that students can practise how gestures are made.

Key words: pragmatics, gestures, non-verbal communication.

\section{La comunicación no verbal: los gestos en ELE}

En la comunicación cara a cara muchas veces es tanto o más importante lo que no se dice como lo que se llega a transmitir con palabras. Esto es, existen toda una serie de estrategias no codificadas lingüísticamente que adquieren un valor comunicativo que resulta esencial para interpretar el mensaje lingüístico y que, en ocasiones, incluso llegan a transmitir significado por ellas mismas, sin que sea necesario contar con un soporte verbal. De hecho, tal es su importancia que en el Diccionario de términos clave de ELE del Instituto Cervantes bajo el lema comunicación no verbal se puede leer lo siguiente:

Todo profesor de lengua extranjera debe enseñar a sus estudiantes los componentes de la comunicación no verbal y las relaciones que se establecen entre los dos sistemas de comunicación. Sobra decir que estos comportamientos varían de una cultura a otra y es imprescindible reconocer estos mecanismos para poder establecer una comunicación fluida con los participantes de la interacción.

Martín Peris (2008)

Tal y como se indica en esta obra, es necesario que los estudiantes de una lengua extranjera se familiaricen con la comunicación no verbal y aprendan a reconocer y a emplear estrategias que les permitan conseguir una comunicarse de forma efectiva. Esta propuesta didáctica se centrará en la enseñanza de gestos, estrategia que forma parte de 
la comunicación no verbal, a través del uso de textos audiovisuales y aplicaciones móviles.

\subsection{Estudios sobre la comunicación no verbal en ELE}

La comunicación no verbal, siguiendo a Cestero, abarca «todos los signos y sistemas de signos no lingüísticos que comunican o se utilizan para comunicar» (1999: 12). En las últimas dos décadas han proliferado los estudios sobre comunicación no verbal en la enseñanza de español para extranjeros. Este tema se ha tratado desde diferentes perspectivas: en propuestas generales (Ardil 2016; Cestero, 1999a 2016; De la Peña y Estévez 1999), en el tratamiento específico de los gestos (Ciarra 2009; Monterubbianesi 2013), en contextos más específicos como el español de los negocios (Fernández-Conde 2009), en contraste entre la cultura hispana y la china (Torres 2010) o a través de propuestas que emplean herramientas específicas como el webquest (Méndez 2016). También se han realizado recopilaciones de signos no verbales (Cestero 1999b) y más concretamente de gestos (Gaviño 2012).

Centrándonos en el tratamiento específico de la enseñanza de gestos, dentro de la propuesta de Cestero, los gestos se definen como «movimientos psicomusculares con valor comunicativo» (1999: 36) y se incluyen dentro del sistema quinésico, uno de los dos sistemas en los que esta autora divide la comunicación no verbal. Desde una perspectiva más metodológica los estudios de Cestero $(1999,2016)$ y Monterubbianesi (2013) proponen, para la enseñanza de gestos, un inventario de fichas para catalogar estos signos no verbales. Estas fichas cuentan con datos tanto sobre el significado y la forma en la que se realiza un gesto como otro tipo de información contextual (pralenguaje, proxémica, cronémica, etc.). Dentro de las propuestas didácticas Ciarra (2009) combina el uso de actividades de rol y el uso de fichas basadas en las introducidas por Cestero (1999) para los gestos más o menos y estar harto de algo. Por su parte, Ardil (2016), para el nivel B1, propone el uso vídeos como método para que los estudiantes identifiquen diferentes gestos. Para trabajar en la producción activa de gestos, emplea actividades basadas en juegos de rol en las que introduce contextos específicos que propician la ejecución de gestos.

\subsection{La importancia de los gestos en la enseñanza de español: MCER y PCIC}

El Marco Común Europeo de Referencia (MCER) propone una manera de entender la enseñanza de lenguas extranjeras que se sitúa más allá de un modelo de lengua estático basado en el estudio de la gramática y el vocabulario. Aprender una lengua significa llegar a poder comunicarse con efectividad, por tanto, se debe incidir tanto en las estrategias codificadas lingüísticamente como en las no codificadas lingüísticamente, es decir, en la comunicación no verbal. Si bien no delimita específicamente en qué nivel ni de qué manera se tiene que tratar la comunicación no verbal, sí incluye una sección dedicada a este sistema, en la que los gestos se enmarcan dentro de las acciones paralingüísticas y, a su vez, dentro del lenguaje corporal. En el nivel de referencia B2, nivel para el que se ha diseñado esta propuesta didáctica, el MCER establece lo siguiente en cuanto a la interacción oral: «[p]uedo participar en una conversación con cierta fluidez $\mathrm{y}$ espontaneidad» (2002: 31). Entendemos que esta fluidez y espontaneidad se manifiesta no solo con mecanismos lingüísticos, sino también con mecanismos paralingüísticos 
como lo son los gestos, puesto que, tal y como se refleja en esta misma obra, «[a]prender a interactuar, por lo tanto, supone más que aprender a comprender y a producir expresiones habladas» (2002: 14). De esta forma, para comunicarse de forma efectiva en español también será necesario aprender, reconocer y utilizar los gestos de manera apropiada.

En el Plan Curricular del Instituto Cervantes (PCIC) señala la importancia de reconocer el papel que tiene la comunicación no verbal en la interacción y, especialmente, de hacer una reflexión sobre la percepción que se tiene sobre esta en las culturas de países hispanohablantes (12. Habilidades y actitudes interculturales. Inventario). Por otra parte, en el apartado 11. Saberes y comportamientos socioculturales la comunicación no verbal se cataloga como comportamiento (relación entre «el contenido y las situaciones de interacción»), si bien no se profundiza posteriormente sobre este concepto. A pesar de que se puede observar que sí tiene en cuenta la comunicación verbal en el PCIC, no parece que se introduzca de manera sistemática la gestualidad. En el nivel B1 (6. Tácticas y estrategias pragmáticas), dentro del subapartado 1.3.1 dedicado a la deixis espacial, se hace referencia a «[a]lusión al lenguaje no verbal» $\mathrm{y}$, de hecho, el ejemplo propuesto incluye los gestos (Él tiene mucho de esto [gesto de dinero]). En el nivel B2, en los apartados 1.4 Pedir confirmación y 4.23. Ofrecerse para hacer algo, se indica que estas acciones también tienen una realización gestual. Por tanto, aunque no se manifieste de forma específica, sí parece indicarse que el nivel B2 es el momento adecuado para introducir la enseñanza de gestos, específicamente de aquellos con los que se expresan acciones de mayor complejidad.

\section{Propuesta práctica para trabajar los gestos en el aula de $\mathbf{E} / \mathbf{L E}$}

Dada la importancia que adquiere la comunicación no verbal en la enseñanza de lenguas extranjeras (Cestero 1999; MCER; PCIC), se ha diseñado una propuesta que tiene como objetivo tratar en clase una de sus manifestaciones, esto es, los gestos. La sesión se centrará en presentar una selección de gestos relacionados con actividades cotidianas y con las emociones. Con esto no solo se pretende que los alumnos reconozcan o puedan reproducir ciertos gestos, sino que también tengan en cuenta que la comunicación se extiende más allá de lo que se codifica lingüísticamente $\mathrm{y}$, de esta manera, contribuir a despertar curiosidad en el alumnado para que tome una mayor conciencia de los gestos que observa en hispanohablantes, de los que ellos mismos hacen, o de los momentos en los que se podrían hacer.

A continuación, se presenta la propuesta diseñada para trabajar los gestos en el aula de ELE basada en el uso de muestras reales de la lengua y de las nuevas tecnologías (aplicaciones para móvil). En primer lugar, se presenta la información general sobre la sesión (temporización, nivel, objetivos y destrezas) y posteriormente se presentan las actividades que se han diseñado para esta. En concreto, se detallan los objetivos y destrezas que se trabajarán y se describen en profundidad los contenidos de cada ejercicio. Se proponen cinco actividades para trabajar los gestos en el aula de ELE. Concretamente, la sesión se estructura en una primera actividad de precalentamiento, dos actividades de andamiaje, una práctica reflexiva y una tarea final en la que los alumnos deberán poner en práctica lo aprendido anteriormente y realizar una serie de gestos para ser grabados. La experiencia práctica completa se puede consultar en el Anexo 1.

Nivel: B2 
Objetivos:

- Reconocer los gestos asociados a las acciones habituales y a los estados de ánimo con los que se relacionan.

- Utilizar, de forma adecuada al contexto y a la emoción que expresen, el lenguaje no verbal.

- Expandir el conocimiento del mundo y la conciencia intercultural del alumnado.

a. Toma de consciencia de los gestos de la cultura de la lengua meta

b. Reconocimiento de las diferencias y similitudes con su cultura de origen.

Destrezas: interacción oral, comprensión audiovisual, expresión escrita

Temporización: 1 hora

\section{Actividad 1}

Tipo de actividad: precalentamiento.

Objetivos:

- Presentar el tema de la sesión

- Activar los conocimientos previos de los alumnos

- Enlazar los conocimientos previos con el tema que se va a tratar

Destrezas: interacción oral

La sesión dará comienzo con una actividad de precalentamiento que servirá, por una parte, para presentar el tema que se va a trabajar, es decir, la gestualidad en español y, por otra parte, para activar los conocimientos o vivencias previas que los alumnos tienen sobre esta cuestión. Se les hará a los alumnos una serie de preguntas para que describan cómo se emplean los gestos en su cultura de origen y qué diferencias han observado entre estos y los gestos que se emplean en español. De esta manera se pretende crear un diálogo en el que participe toda la clase que permita introducir el tema a través de una reflexión conjunta.

\section{Actividad 2}

Tipo de actividad: andamiaje

Objetivos:

- Reconocer a través del contexto gestos relacionados con acciones cotidianas.

Destrezas: expresión oral, comprensión audiovisual, expresión escrita

Tras contextualizar el tema de la sesión, se introducirá la primera actividad de andamiaje. Se trabajará con un sketch del programa de humor Splunge emitido en Televisión Española. En este vídeo, aparecen una serie de gestos relacionados con acciones de la

\footnotetext{
${ }^{1}$ https://es.wikipedia.org/wiki/Splunge
} 
vida cotidiana (pedir la cuenta, pedir una caña, cocinar, tener frío, etc.). Se llevará a cabo un primer visionado en el que se les pedirá a los alumnos que describan qué está ocurriendo en esta escena. De esta manera, se comprobará que han entendido el tema del vídeo y se aclararán las dudas que puedan surgir. A continuación, completarán los espacios vacíos de la parte final de la transcripción del vídeo que representan diferentes gestos relacionados con acciones habituales que pueden identificarse por el contexto. Para esta tarea, se realizarán dos o tres visionados del fragmento del vídeo transcrito. Finalmente, se corregirá la actividad de manera conjunta y se irá viendo en detalle la realización de cada gesto y su significado.

\section{Actividad 3}

Tipo de actividad: andamiaje

Objetivos:

- Reconocer a través del contexto gestos relacionados con las emociones.

Destrezas: expresión oral, comprensión audiovisual

Este ejercicio constituye la segunda actividad de andamiaje. En este caso, se trabajará con gestos ligados a las emociones (vergüenza, sorpresa, preocupación, etc.) y se empleará para ello material audiovisual extraído del programa de telerrealidad Gandía Shore. Se han seleccionado nueve vídeos de corta duración en los que los hablantes representan un gesto que expresa una emoción. Los alumnos deberán adivinar la emoción que se representa mediante gestos en cada uno de los vídeos. Cabe señalar que en ocasiones se han escogido gestos que expresan la misma emoción de manera diferente, así como gestos similares que expresan emociones diferentes. Esta decisión se ha tomado de forma consciente con el fin de tratar con mayor profundidad la necesidad de tener en cuenta el contexto para poder interpretar correctamente una situación (véase la actividad 4).

\section{Actividad 4}

Tipo de actividad: Práctica reflexiva sobre las dos actividades anteriores

Objetivos:

- Repasar los gestos ligados a las emociones trabajados en la actividad anterior

- Reflexionar sobre la representación de los gestos: las similitudes entre gestos diferentes y las diferentes maneras de expresar una emoción

Destrezas: interacción oral, expresión escrita

En esta actividad se reforzarán los contenidos vistos en la actividad anterior y se reflexionará sobre ellos. Partiendo de las emociones trabajadas previamente, se tratarán las diferentes maneras que se han visto de representarlas mediante gestos. Por otra parte, se examinarán gestos que se representan de forma similar (taparse la cara con las manos para expresar vergüenza o preocupación, por ejemplo) y se discutirá cómo, en caso de ambigüedad, se puede proceder para reconocer la emoción que expresa el gesto (factores contextuales, la expresión de hablante, etc.). Finalmente, los alumnos deberán realizar una descripción por escrito de la ejecución de cada gesto que se ha trabajado en la actividad anterior.

\section{Actividad 5}

Tipo de actividad: Tarea final 
Objetivos:

- Poner en práctica los gestos aprendidos

- Practicar la toma de decisiones y la negociación

Destrezas: interacción oral

Por último, los alumnos llevarán a cabo la tarea final. En este ejercicio los alumnos escogerán un gesto que exprese una emoción (trabajados en las actividades 3 y 4) y deberán grabarse representándolo. Si la clase es muy numerosa, se dividirá a los alumnos en grupos o parejas y cada grupo trabajará un gesto. Si, por el contrario, se cuenta con una clase con un número reducido de estudiantes se trabajará individualmente. Antes de ser grabados, los alumnos deberán representar el gesto ante la clase o dentro del grupo, quienes actuarán de jueces y decidirán si la ejecución se ajusta a cómo se realiza el gesto. Una vez quede aprobado el gesto, el profesor se encargará de grabar a los alumnos. Se utilizará para ello preferentemente una aplicación para móvil que permita grabar gifs ${ }^{2}$ (en nuestro caso se usó la aplicación gratuita GIPHY Cam). El uso de este formato resulta adecuado para nuestros propósitos, puesto que se producen archivos que pueden compartirse fácilmente con los alumnos. Otra de sus ventajas reside precisamente en la reproducción en bucle, lo que permite poder ver la ejecución de un gesto tantas veces como se quiera sin tener que volver a iniciar la reproducción cada vez que acabe.

\section{Pilotaje de la propuesta}

La propuesta presentada en este artículo se pilotó en un grupo de siete estudiantes de español como lengua extranjera, que están en un contexto de inmersión realizando un curso de gramática española en la Universitat de València. El uso de fragmentos extraídos de programas de humor y de telerrealidad resulta atractivo para el alumnado puesto que representan muestras reales de español. Además, tratándose de un público joven en el caso del pilotaje de la propuesta, los contenidos se adaptaron a sus gustos. La enseñanza a partir de materiales audiovisuales y especialmente a través del uso de nuevas tecnologías como las aplicaciones para móvil contribuyó a la dinamización de la sesión, puesto que las alumnas fueron capaces de implicarse de forma más directa con los contenidos trabajados y de motivarse a través de vídeos que les resultaron afines. En general, se puede afirmar que la puesta en práctica de la propuesta aquí presentada tuvo una buena recepción por parte de las alumnas.

Alguno de los aspectos que hay que tener en cuenta a la hora de poner en práctica esta propuesta son, por una parte, el número de alumnos de los que consta cada clase. En clases más numerosas, sería necesario un reajuste en la temporización de la sesión, puesto que es posible que se necesite más tiempo para llevarla a cabo, y también en el planteamiento de algunos ejercicios. La actividad 4 se puede hacer de formar conjunta si el número de alumnos es reducido, mientras que si se cuenta con un grupo más grande se podría optar por que los alumnos trabajen en grupo en la descripción de los gestos.

Otro aspecto que puede influir en el desarrollo de esta propuesta es el carácter de los alumnos. En la última actividad los estudiantes tienen que ser grabados realizando un gesto, lo que puede causar que algunos alumnos se sientan incómodos o que incluso se

\footnotetext{
${ }^{2}$ Los gifs son archivos con una extensión propia (.gif) que reproducen en bucle vídeos cortos sin sonido.
} 
nieguen a participar en esta tarea. En casos en los que alguien no desee actuar delante de una cámara, se le podrá asignar otro papel en la actividad como, por ejemplo, puede ser que participe de manera más activa como juez evaluando los gestos de ser grabados.

Por último, también se podría plantear esta actividad como punto de partida para realizar un proyecto de mayor envergadura en el que se trabajen los gestos durante todo un curso. Esto es especialmente adecuado para cursos de español extensivos en contextos de inmersión. Esta primera sesión se plantearía como introductoria en el tema de la gestualidad en español para después pedir a los alumnos que una vez a la semana o cada dos semanas uno de ellos eligiera un gesto sobre el que profundizar. El alumno deberá, por tanto, estudiarlo y exponer en clase su significado y la forma de realizarlo y posteriormente grabarse para así ir creando conjuntamente un pequeño diccionario de gestos interpretados por los propios alumnos del curso de español.

\section{Conclusiones}

Se presenta una propuesta para llevar al aula de ELE una parcela de la comunicación no verbal como son los gestos. Se pretende no solo que los alumnos aprendan a reconocer y a ejecutar los gestos que se han estudiado específicamente en la sesión, sino que también tomen conciencia de la importancia que la gestualidad en la interacción para comunicar tanto acciones como emociones. De esta manera, se espera que el alumnado empiece a desarrollar una sensibilidad para detectar los gestos con los que se vaya encontrado y que pueda empezar a emplearlos de forma autónoma y espontánea.

Consideramos, además, que esta propuesta puede adaptarse fácilmente a otros niveles, lo que permite trabajar un elemento tan fundamental para la interacción como la gestualidad en todas las etapas del aprendizaje del español. En los niveles más bajos se puede limitar a trabajar los gestos ligados a acciones cuotidianas o trabajar de forma más guiada los gestos que expresan emociones. Por otra parte, en niveles más avanzados, se puede profundizar sobre los matices que aportan los gestos en la interacción cara a cara o sobre cómo y cuándo usarlos. A modo de conclusión, los resultados de la primera puesta en práctica muestran que es factible trabajar el desarrollo de los gestos en el aula de ELE a partir de material audiovisual real y de aplicaciones móviles. El pilotaje de la propuesta, además de probar su viabilidad, demuestran que ha supuesto una actividad recibida positivamente por las alumnas y que ha conseguido despertar su interés y fomentar su implicación en las tareas.

\section{Bibliografía}

ARDIL GonZÁlez, Ana Fuensanta (2016). El lenguaje no verbal. Claves culturales para la competencia comunicativa e intercultural. Universidad de Oviedo: Trabajo de fin de Máster.

Cestero Manceda, Ana María (1999a). Comunicación no verbal y enseñanza de lenguas extranjeras. Madrid: Arco/Libros.

Cestero Manceda, Ana María (1999b). Repertorio básico de signos no verbales del español. Madrid: Arco/Libros.

Cestero MancedA, Ana María (2016). «La Comunicación no verbal: propuestas 
metodológicas para su estudio». Linred, 13.

CiARra TejadA, Alzine (2009). El lenguaje no verbal. utilidad y dificultades de su enseñanza en el aula de E/LE. Reflexión y propuesta didáctica. Universidad de Salamanca: Trabajo de final de Máster.

CONSEJO DE EUROPA (2002). Marco común europeo de referencia para las lenguas: aprendizaje, enseñanza, evaluación. Madrid: Ministerio de Educación, Cultura y Deporte, Instituto Cervantes, Anaya.

De la PeÑa Portero, Alicia y María Estévez Funes (1999). «Hablar sin palabras: la comunicación no verbal en clase de E/LE». En M. Franco, C. Soler, J. de Cos, M. Rivas, F. Ruiz (eds.); Nuevas perspectivas en la enseñanza del español como lengua extranjera (pp. 519-525). Cádiz: Servicio de publicaciones de la Universidad de Cádiz.

FERNÁNDEZ-CONDE RODRÍGUEZ, Manuel (2009). «La enseñanza de la comunicación no verbal en la clase de español para los negocios». Marcoele, 8.

GaviÑo RodríGUEZ, Victoriano (2012). Diccionario de Gestos Españoles. Disponible en: http://www.coloquial.es/es/diccionario-de-gestos-espanoles/

Instituto Cervantes (2006). Plan Curricular del Instituto Cervantes. Madrid: Instituto Cervantes-Biblioteca Nueva.

MARTín Peris, E. et. al. (2008). Diccionario de términos clave de ELE. Madrid: SGEL. Disponible en: http://cvc.cervantes.es/ensenanza/biblioteca_ele/diccio_ele/indice.htm

MÉNDEZ GUERRERO, Beatriz (2016). «El componente no verbal en el aula de ELE. Consideraciones para su aprendizaje significativo e intercultural a partir de WebQuest». Revista Nebrija de Lingüística Aplicada a la Enseñanza de Lenguas, 20.

MONTERUBBIANESI, Maria Giovanna (2013). «La comunicación no verbal en el aula: una propuesta de clasificación y análisis de los gestos para las clases de E/LE». Marcoele, 16.

TORRES SÁNCHEZ, Amparo (2010). «Los signos no verbales en clase de ELE». SinoELE [suplemento], Actas de las III Jornadas de Formación de Profesores de E/LE en China: Integraciones de destrezas comunicativas en el aula de ELE en China: dificultades y propuestas de aplicación. 


\section{ANEXO 1}

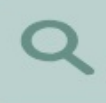

Dime cómo te mueves y te diré qué expresas

Gloria Uclés Ramada Universitat de València Val.Es.Co XIV Foro ELE

En esta sesión vamos a trabajar la comunicación no verbal; en concreto, la gestualidad. En español, los gestos se utilizan con gran frecuencia para expresar acciones, reforzar lo dicho, así como para comunicar estados de ánimo.

1.¿Qué sabes sobre la gestualidad en español? ¿Cuál ha sido tu experiencia con ellos? ¿Hay muchas diferencias con los gestos que usas en tu lengua de origen?

2.Vamos a ver un sketch humorístico sobre la comunicación no verbal.

a.Explica brevemente cuál es el tema del vídeo.

b. Rellena los huecos de la transcripción del final del vídeo con una palabra que crees que está representando el gesto que se hace.

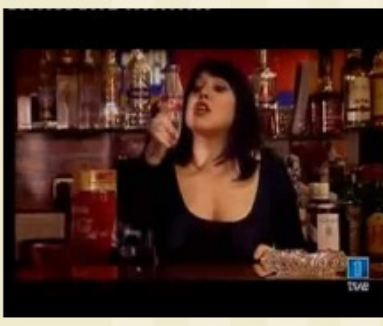

\section{Camarera: Bueno, a ver, a ver. Lo que sí les pido es un poco de} ¿eh?

Cuando yo termine de le llevo a usted su

llevo a usted su que tiene un poquito de
están ustedes todos y después bajo un poquito el pagar con
chmm?

¿No es así?

$Y$ luego le llevo a usted sus dos__ porque no tienen

Clientes: Sí, sí, sí. y tampoco tienen que

Camarera: ¿Lo ven como hablando se entiende la $?$

3. A continuación, veremos 9 fragmentos cortos sin sonido en el que se muestran uno o varios gestos. Identifica a qué estado de ánimo pueden corresponder.

Vídeo 1:

Vídeo 4:

Vídeo 2:

Vídeo 3:

Vídeo 7

Vídeo 5:

Vídeo 8:

Vídeo 6

Vídeo 9:

4. ¿Qué gestos has detectado para expresar las emociones de la actividad anterior? ¿Son todos iguales? ¿Se puede expresar una emoción con diferentes gestos? ¿Puede un gesto expresar diferentes emociones?

\section{Dime cómo te mueves y te diré qué expresas}

5. ¡Ahora tú! Individualmente o en grupos, vamos a escoger uno de gestos de las actividades anteriores. A continuación, practicaremos el gesto $y$, finalmente, lo grabaremos para que forme parte de nuestro pequeño diccionario de gestos.
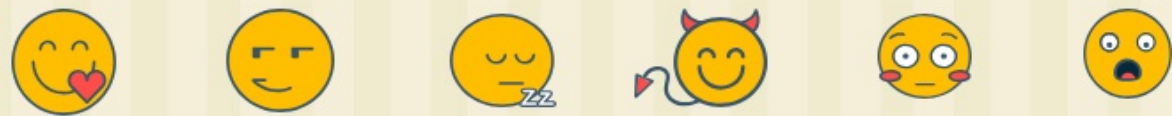